\title{
QCD SPECTRAL SUM RULES AND SPONTANEOUSLY BROKEN CHIRAL SYMMETRY *
}

\author{
E. Marco and W. Weise \\ Physik-Department, \\ Technische Universität München, \\ D-85747 Garching, Germany
}

September 15, 2018

\begin{abstract}
The gap $\Delta=4 \pi f_{\pi} \simeq 1.2 \mathrm{GeV}$ of spontaneous chiral symmetry breaking is introduced as a scale delineating resonance and continuum regions in the QCD spectral sum rules for vector mesons. Basic current algebra results are easily recovered, and accurate sum rules for the lower moments of the spectral distributions are derived. The in-medium scaling of vector meson masses finds a straightforward interpretation, at least in the narrow width limit.
\end{abstract}

PACS: 11.30.Qc; 11.55.Hx

${ }^{*}$ Work supported in part by BMBF, GSI and Conselleria de Cultura, Educació i Ciència de la Generalitat Valenciana. 
Spectral sum rules have frequently been used to connect observable information through dispersion relations with the operator product expansion (OPE) in QCD, in the form of either SVZ sum rules [四] or finite energy sum rules (FESR) 22, 3, 4]. Prototype examples are the sum rules for the lightest $(\rho$ and $\omega)$ vector mesons. The starting point is the vacuum current-current correlation function,

$$
\begin{aligned}
\Pi_{\mu \nu}(q) & =i \int d^{4} x e^{i q \cdot x}\left\langle 0\left|\mathcal{T} j_{\mu}(x) j_{\nu}(0)\right| 0\right\rangle \\
& =\left(g_{\mu \nu}-\frac{q_{\mu} q_{\nu}}{q^{2}}\right) \Pi\left(q^{2}\right)
\end{aligned}
$$

where $\mathcal{T}$ denotes the time-ordered product and the currents are specified, for the case of interest here, as $j_{\mu}^{(\rho)}=\left(\bar{u} \gamma_{\mu} u-\bar{d} \gamma_{\mu} d\right) / 2$ for the $\rho$ channel and $j_{\mu}^{(\omega)}=\left(\bar{u} \gamma_{\mu} u+\bar{d} \gamma_{\mu} d\right) / 6$ for the $\omega$ channel. We work as usual with the spectrum

$$
R\left(q^{2}\right)=-\frac{12 \pi}{q^{2}} \operatorname{Im} \Pi\left(q^{2}\right)
$$

normalized to the ratio $\sigma\left(e^{+} e^{-} \rightarrow\right.$ hadrons $) / \sigma\left(e^{+} e^{-} \rightarrow \mu^{+} \mu^{-}\right)$.

The nuclear physics interest in QCD sum rules is motivated by applications of SVZ type Borel sum rules not only in vacuum, but also in nuclear matter in order to extract in-medium properties of vector mesons [5, 6]. The commonly adopted procedure is to use a schematic "duality" ansatz for $R$, with the vector meson resonance represented as a $\delta$-function and a step function continuum starting at a threshold $s_{0}$. The position of the resonance and the threshold $s_{0}$ are then fitted by requiring consistency with the vacuum or in-medium OPE side of the sum rule.

The continuum threshold $s_{0}$ is usually introduced as a free parameter. On the other hand, spontaneous chiral symmetry breaking in QCD suggests that the mass gap which separates the QCD ground state from the high energy continuum should be expressed as some multiple of the pion decay constant, $f_{\pi}=92.4 \mathrm{MeV}$, since this is the only remaining scale in the limit of vanishing quark masses.

In the present paper we propose to identify this continuum threshold with the chiral gap parameter, $\Delta=4 \pi f_{\pi} \simeq 1.2 \mathrm{GeV}$, by setting

$$
\sqrt{s_{0}}=\Delta=4 \pi f_{\pi}
$$


and thereby unifying QCD sum rules with spontaneous chiral symmetry breaking.

Starting from this assumption we shall demonstrate that QCD spectral sum rules combined with vector meson dominance (VMD) immediately imply well known current algebra relations, a very welcome feature. When realistic spectral distributions are used, the transition between resonance region and continuum is no longer sharp, but the chiral mass gap (3) is shown still to control the smooth turnover from the hadronic part to the asymptotic QCD domain of the spectrum for both $\rho$ and $\omega$ channels. We also point out briefly that our hypothesis (3) permits one to understand the in-medium results of ref. [5] in a simple and straightforward way, using the leading density dependence of the pion decay constant.

Our approach is based on rigorous sum rules for the lowest moments of the spectral distribution (2). A direct access to these moment sum rules is best given by the FESR method [2, 3, 0]. Consider the vacuum correlation function $\Pi\left(q^{2}=s\right)$ of Eq. (1) in the complex $s$-plane where it has a cut along the positive real axis. Choose a closed loop $\gamma$ consisting of a path which surrounds and excludes the cut along Res $>0$, and joins with a circle $C_{s_{0}}$ of fixed radius $s_{0}$. Cauchy's theorem implies $\oint_{\gamma} d s s^{N-1} \Pi(s)=0$ for integer $N \geq 0$. Separating this integral and using Eq. (2) gives

$$
\int_{0}^{s_{0}} d s s^{N} R(s)=-6 \pi i \oint_{C_{s_{0}}} d s s^{N-1} \Pi(s)=6 \pi s_{0}^{N} \int_{0}^{2 \pi} d \theta e^{i N \theta} \Pi\left(s_{0} e^{i \theta}\right) .
$$

It remains to evaluate the r.h.s. integral along the circle of radius $s_{0}$. For sufficiently large $s_{0}$ one can use perturbative QCD and add non-perturbative corrections via the OPE:

$$
\Pi(s)=\Pi^{\mathrm{pQCD}}(s)+\frac{d}{12 \pi^{2}} \sum_{n \geq 0}(-)^{n} \frac{c_{n+1}}{s^{n}},
$$

with $d=3 / 2$ or $1 / 6$ for the $\rho$ or $\omega$ channels, respectively. The parameters $c_{n}$ have dimension (mass $)^{2 n}$, with $c_{1}=-3\left(m_{u}^{2}+m_{d}^{2}\right)$ and the dimension-4 condensates $c_{2}=\left(\pi^{2} / 3\right)\left\langle\left(\alpha_{s} / \pi\right) G_{\mu \nu} G^{\mu \nu}\right\rangle+4 \pi^{2}\left\langle m_{u} \bar{u} u+m_{d} \bar{d} d\right\rangle$ which are reasonably well under control. The $c_{3}$ involves the (much less certain) four-quark condensates. In practice $c_{1}$ is negligibly small, and the quark condensate piece in $c_{2}$ can be dropped in comparison with the gluon condensate term. As usual, we ignore logarithmic corrections to the condensates. 
The pQCD part of $\Pi(s)$ is calculated to third order on $\alpha_{s}$ using the $\overline{M S}$ scheme [8]. The result is

$$
\Pi^{\mathrm{pQCD}}(s)=\frac{d}{12 \pi^{2}} \sum_{n=0}^{3}\left(\frac{\alpha_{s}\left(\mu^{2}\right)}{\pi}\right)^{n} \Pi^{(n)}\left(s ; \mu^{2}\right)
$$

at a renormalization point $\mu^{2}$, with $\Pi^{(0)}=s\left[K_{0}+\ln \left(-s / \mu^{2}\right)\right]$ and $\Pi^{(n)}=$ $s\left[K_{n}+\sum_{m=1}^{n} A_{m n} \ln ^{m}\left(-s / \mu^{2}\right)\right]$ for $n=1,2,3$. The constants $K_{n}$ are irrelevant since they drop out in the loop integral (画). The relevant coefficients (for $N_{f}=3$ flavours) of the logarithmic terms are $A_{11}=1, A_{12}=1.641$, $A_{22}=-1.125, A_{13}=-10.28, A_{23}=-5.69, A_{33}=1.69$. The renormalization point can be chosen at $\mu^{2}=s_{0}$.

Inserting $\Pi\left(s_{0} e^{i \theta}\right)$ from Eqs. (5, 6) and using $\ln \left(-e^{i \theta}\right)=i(\theta-\pi)$, the r.h.s. integral of Eq. (4) is easily worked out and one arrives at the following set of sum rules for the lowest spectral moments with $N=0,1,2$ :

$$
\int_{0}^{s_{0}} d s s^{N} R(s)=d\left[\frac{s_{0}^{N+1}}{N+1}\left(1+\delta_{N}\right)+(-)^{N} c_{N+1}\right] .
$$

The perturbative QCD corrections up to $O\left(\alpha_{s}^{3}\right)$ are summarized as

$$
\begin{aligned}
\delta_{N}= & \frac{\alpha_{s}}{\pi}+\left(\frac{\alpha_{s}}{\pi}\right)^{2}\left[A_{12}-\frac{2}{N+1} A_{22}\right] \\
& +\left(\frac{\alpha_{s}}{\pi}\right)^{3}\left[A_{13}-\frac{2}{N+1} A_{23}+\left(\frac{6}{(N+1)^{2}}-\pi^{2}\right) A_{33}\right],
\end{aligned}
$$

where $\alpha_{s}$ is taken at $\mu^{2}=s_{0}$. Note that different $\delta_{N}$ apply for the various moments of $R(s)$, and that condensates of different mass dimension appear well separated in the different moments. For example, uncertain four-quark condensates enter only at $N=2$, whereas the moments with $N=0,1$ are free of such uncertainties. It can be readily demonstrated that the results, Eqs. (7,8), are rigorously consistent with those obtained using the Borel sum rule method. It is also interesting to note that our deduction of the sum rules (7) is analogous to the procedure used to extract $\alpha_{s}$ from $\tau$ decays [9].

Before applying these sum rules to realistic spectral distributions, we turn first to a schematic model for $R(s)$ which combines vector meson dominance (VMD) and the QCD continuum:

$$
R_{V}(s)=12 \pi^{2} \frac{m_{V}^{2}}{g_{V}^{2}} \delta\left(s-m_{V}^{2}\right)+\text { continuum } \quad(V=\rho, \omega),
$$


with $g_{\rho}=g_{\omega} / 3=g$, the universal vector coupling constant. For convenience we discuss the $\rho$ meson sum rules first. We set $s_{0}=16 \pi^{2} f_{\pi}^{2}$ according to our conjecture (3) and find the sum rules for the first two moments:

$$
\begin{gathered}
\int_{0}^{s_{0}} d s R_{\rho}(s)=12 \pi^{2} \frac{m_{\rho}^{2}}{g^{2}}=\frac{3}{2}\left(4 \pi f_{\pi}\right)^{2}\left(1+\delta_{0}\right)+\frac{3}{2} c_{1}, \\
\int_{0}^{s_{0}} d s s R_{\rho}(s)=12 \pi^{2} \frac{m_{\rho}^{4}}{g^{2}}=\frac{3}{4}\left(4 \pi f_{\pi}\right)^{4}\left(1+\delta_{1}\right)-\frac{3}{2} c_{2} .
\end{gathered}
$$

Once the hypothesis (3) is launched, there are no free parameters in these sum rules. Dropping the QCD corrections for the moment, the sum rule (10) immediately gives

$$
m_{\rho}^{2}=2 g^{2} f_{\pi}^{2},
$$

the well-known KSFR relation [10], while the sum rule (11) for the first moment further specifies

$$
g=2 \pi
$$

The results (12,13) are quite remarkable: identifying the onset of the continuum spectrum with the gap $\Delta=4 \pi f_{\pi}$, the scale for spontaneous chiral symmetry breaking, a unification of QCD spectral sum rules with current algebra emerges, yielding $m_{V}=\sqrt{8} \pi f_{\pi}=\Delta / \sqrt{2}$ in leading order (identical relations hold for both $\rho$ and $\omega$ meson). The condition $g=2 \pi$ is actually consistent with the effective action of the $S U(3) \times S U(3)$ non-linear sigma model and the Wess-Zumino term. Application of the QCD corrections moves $g$ to within less than $10 \%$ of the empirical $g_{\rho} \simeq 5.04$ deduced from the $\rho \rightarrow e^{+} e^{-}$decay width.

Let us now turn from schematic to realistic spectral distributions. The vector mesons have energy dependent widths from their leading decay channels $\rho \rightarrow \pi \pi$ and $\omega \rightarrow 3 \pi$, etc. Multipion $(n \pi)$ channels (with $n$ odd/even for $I=0,1)$ open up and continue toward the asymptotic pQCD spectrum. It would then seem difficult, at first sight, to locate the gap $\Delta$ which delineates the resonance from the QCD continuum. Remarkably, though, the turnover from resonance to continuum is still governed by the scale set by the chiral gap $\Delta=4 \pi f_{\pi}$. One can simply replace the sharp edge at $s_{0}=\Delta^{2}$ by a smooth interpolation in the interval $\Delta^{2}-0.6 \mathrm{GeV}^{2} \lesssim s \lesssim \Delta^{2}+0.6 \mathrm{GeV}^{2}$. 


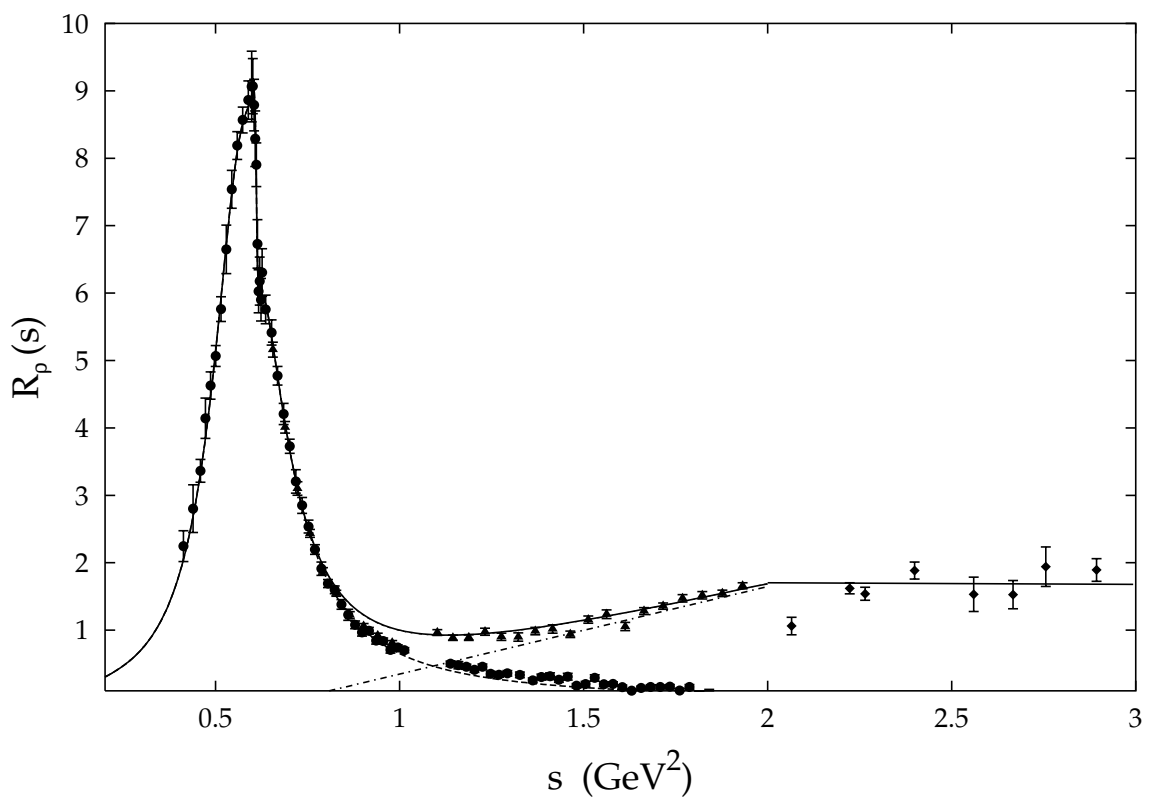

Figure 1: Spectral distribution $R_{\rho}(s)$ in the isovector ( $\rho$ meson) channel. Dashed line: resonant part; dot-dashed line: linear interpolation centred at $\Delta^{2}=\left(4 \pi f_{\pi}\right)^{2}$; solid line: sum of resonance and continuum contributions. Experimental data: full dots from $e^{+} e^{-} \rightarrow \pi^{+} \pi^{-}$[12]; triangles [13] and diamonds [14] from total $e^{+} e^{-} \rightarrow n \pi$ with $n$ even.

In essence, the spreadings of the resonance and the gap edge amount to incorporating $1 / N_{c}$ corrections to the zero width spectrum (9).

In practice the calculation proceeds as follows. Consider the $\rho$ meson first. The resonant part of the spectrum is well described using effective field theory as shown in ref. [6], including $\rho \omega$ mixing. Guided by this approach we use a parametrized form, given in Ref. [11], which reproduces the $e^{+} e^{-} \rightarrow \pi^{+} \pi^{-}$ data (see dashed curve in Fig. 1). We let the QCD continuum start at $s_{c}=2$ $\mathrm{GeV}^{2}$ and use a linear interpolation across the interval $0.8 \mathrm{GeV}^{2} \lesssim s \lesssim s_{c}$ centered at $\Delta^{2}$ (dashed-dotted curve in Fig. 1). When added to the tail of the $\rho$ resonance this interpolation obviously works well in reproducing the total $e^{+} e^{-} \rightarrow 2 \pi, 4 \pi, \ldots$ data in the $I=1$ channel. We now employ the sum rules (7) with $s_{0}=s_{c}$ and check overall consistency, using $\alpha_{s}\left(s_{0}\right)=0.39$. For the lowest moment with $N=0$ the left-hand side gives $\int_{0}^{s_{c}} d s R_{\rho}(s)=3.527$ $\mathrm{GeV}^{2}$, while the right-hand side gives $3.521 \mathrm{GeV}^{2}$. For $N=1$ the l.h.s. 


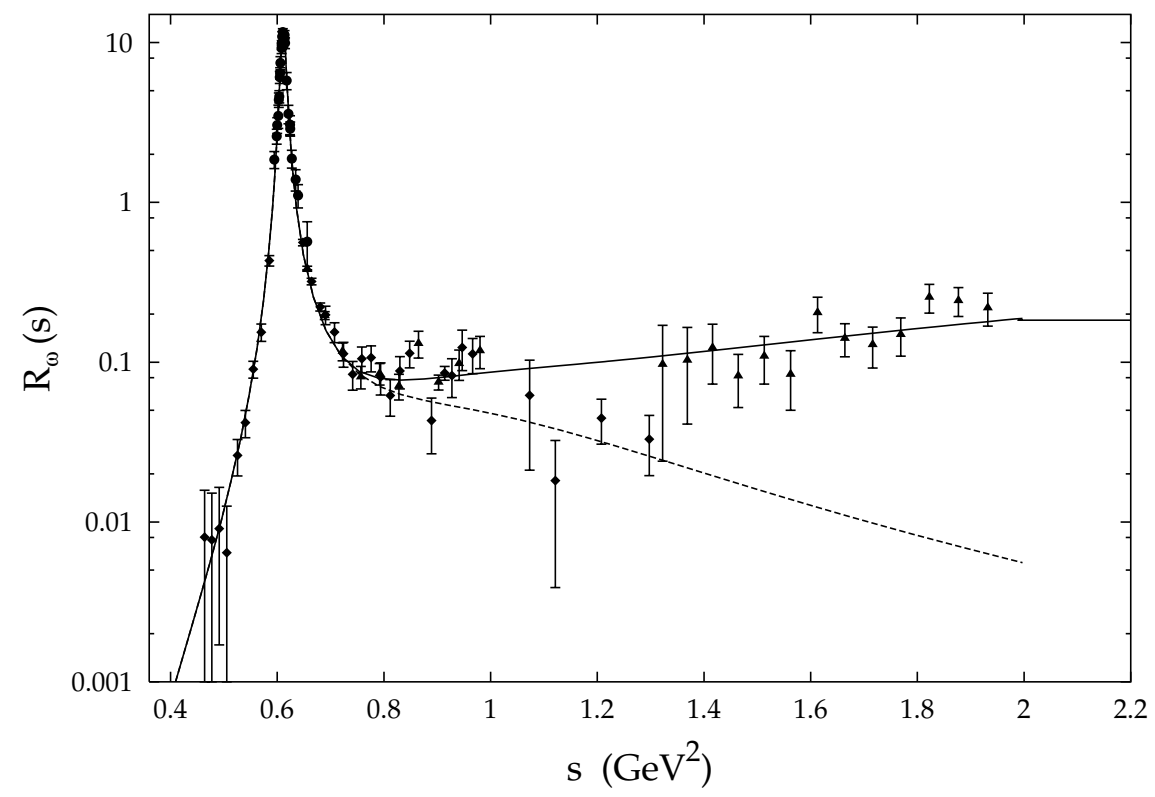

Figure 2: Spectral distribution $R_{\omega}(s)$ in the isoscalar ( $\omega$ meson) channel. Dashed curve: resonant part; solid curve: sum of resonance and continuum contributions. Experimental data: dots [12]; diamonds and triangles [13], from $e^{+} e^{-} \rightarrow n \pi$ with $n$ odd.

integral gives $\int_{0}^{s_{c}} d s s R_{\rho}(s)=3.27 \mathrm{GeV}^{4}$, while the r.h.s. using a gluon condensate $\left\langle\left(\alpha_{s} / \pi\right) G^{2}\right\rangle=(0.36 \mathrm{GeV})^{2}$ yields $3.32 \mathrm{GeV}^{4}$, so there is consistency to better than $2 \%$. The second moment $(N=2)$ involves uncertain four-quark condensates and is more sensitive to the detailed form of the spectrum at higher energies. Its discussion will be delegated to a forthcoming paper, but at this point we can already conclude that the usual factorization ansatz for the four-quark condensate assuming ground state dominance turns out not to be justified: factorization underestimates the four-quark condensate by a large amount. The statements about the low $(N=0,1)$ spectral moments are of course free of such uncertainties.

For the $\omega$ meson spectrum we are again guided by the effective Lagrangian approach (Ref. [6]). The resonant part is parametrized as in Ref. [15]. (We stay in the $u, d$-quark sector and therefore omit $\omega \phi$ mixing). Otherwise we follow a scheme analogous to that for the $\rho$ meson. The QCD continuum starts at $s_{c}=2 \mathrm{GeV}^{2}$ and the same (linear) interpolation around $s=\Delta^{2}$ is added to the resonance tail (dashed curve in Fig. 2) between 0.8 and $2 \mathrm{GeV}^{2}$. 
The result (Fig. 2) compares quite well with the data in the $I=0 e^{+} e^{-} \rightarrow$ hadrons channel, though with admittedly poor statistics in the region above the $\omega$ resonance. For the lowest spectral moment we find $\int_{0}^{s_{c}} d s R_{\omega}(s)=$ $0.3917 \mathrm{GeV}^{2}$ as compared to $0.3912 \mathrm{GeV}^{2}$ from the r.h.s. of the $N=0$ sum rule (7). The $N=1$ moment gives $\int_{0}^{s_{c}} d s s R_{\omega}(s)=0.371 \mathrm{GeV}^{4}$, in perfect agreement with the r.h.s. value $0.369 \mathrm{GeV}^{4}$. Consistency of the second moment requires the same four-quark condensate as observed for the $\rho$ meson, substantially larger than the value suggested by factorization into $\langle\bar{q} q\rangle^{2}$.

In summary, the degree of consistency found in this approach is quite impressive, at least for the first two moments of the spectral distributions. In particular, the crossover between $\rho$ and $\omega$ resonance regions and the asymptotic continuum, although smooth, is still controlled by the chiral symmetry breaking scale $\Delta=4 \pi f_{\pi}$.

With this observation in mind we can briefly comment on the in-medium version of the schematic model (9), with $m_{V}$ replaced by density dependent masses $m_{V}^{*}(\rho)$ (for $\left.V=\rho, \omega\right)$ and the continuum onset $s_{0}$ replaced by $s_{0}^{*}(\rho)$. Using such a parametrization Hatsuda and Lee found $m_{V}^{*} \simeq m_{V}\left(1-0.16 \rho / \rho_{0}\right)$ in their in-medium QCD sum rule analysis [5]. This result can now be given a straightforward interpretation. The nuclear matter analogue of the sum rules (10, 11) gives the leading behaviour $m_{V}^{*}(\rho) \simeq \sqrt{8} \pi f_{\pi}^{*}(\rho)$, where $f_{\pi}^{*}$ is the pion decay constant in matter related to the time-component of the axial current. The in-medium PCAC relation $f_{\pi}^{* 2}=\left(m_{q} / m_{\pi}^{2}\right)\langle\bar{q} q\rangle^{*}+\cdots$ implies that $f_{\pi}^{*}$ scales like the square root of the quark condensate to leading chiral order. The magnitude of this condensate at nuclear matter density $\rho_{0}$ (see [16] for a review and further references) is expected to drop by about $1 / 3$ of its value at $\rho=0$, so $m_{V}^{*}\left(\rho_{0}\right) / m_{V} \simeq f_{\pi}^{*}\left(\rho_{0}\right) / f_{\pi} \simeq 5 / 6$ in the zero width limit. The same result is also found in ref. [6] and in Brown-Rho scaling [17]. The $\omega$ meson which is predicted still to survive as a reasonably narrow state in nuclear matter [G], should be a good indicator of the way in which the chiral gap decreases with increasing density. The much broader $\rho$ meson, on the other hand, is probably not very useful for this purpose [6].

In conclusion, introducing the chiral gap $\Delta=4 \pi f_{\pi}$ as a relevant scale in QCD spectral sum rules for vector mesons establishes important connections with current algebra and the in-medium scaling of vector meson masses. Further work along these lines is in progress. 


\section{References}

[1] M.A. Shifman, A.I Vainshtein and Z.I. Zakharov, Nucl. Phys. B 147 (1979) 385, 448.

[2] K.G. Chetyrkin, N.V. Krasnikov and A.N. Tavkhelidze, Phys. Lett. B 76 (1978) 83.

[3] J. Fischer and P. Kolár, Z. Phys. C 34 (1987) 375.

[4] F. Klingl and W. Weise, Eur. Phys. J. A 4 (1999) 225.

[5] T. Hatsuda and S.H. Lee, Phys. Rev. C 46 (1992) 34.

[6] F. Klingl, N. Kaiser and W. Weise, Nucl. Phys. A 424 (1997) 527.

[7] V. Giménez, J. Bordes and J. Peñarrocha, Nucl. Phys. B 357 (1991) 3.

[8] S.G. Gorishny, A.L. Kataev and S.A. Larin, JETP Lett. 53 (1991) 127.

[9] A. Pich, Nucl. Phys. B (Proc. Suppl.) 39 B (1995) 326.

[10] K. Kawarabayashi and M. Suzuki, Phys. Rev. Lett. 16 (1996) 255; Riazuddin and Fayyazuddin, Phys. Rev. 147 (1966) 1071.

[11] M. Benayoun et al., Z. Phys. C 58 (1993) 31.

[12] L.M. Barkov et al., Nucl. Phys. B 256 (1985) 365.

[13] S.I. Dolinsky et al., Phys. Rep. 202 (1991) 99.

[14] C. Bacci et al., Nucl. Phys. B 184 (1981) 31.

[15] N.N. Achasov and A.A. Kozhevnikov, Phys. Rev. C 57 (1998) 4334.

[16] W. Weise, Les Houches Lectures, Session LXVI in: Trends in Nuclear Physics, 100 Years Later, Elsevier, Amsterdam (1998), p. 423.

[17] G.E. Brown and M. Rho, Phys. Rev. Lett. 66 (1991) 2720. 